\title{
Preventing chemotherapy-induced nausea and vomiting in patients with lung cancer: efficacy of NEPA (netupitant-palonosetron), the first combination antiemetic
}

\author{
Paul J. Hesketh ${ }^{1}$ - Marco Palmas ${ }^{2}$ - Pierre Nicolas ${ }^{3}$ \\ Received: 29 March 2017 / Accepted: 16 October 2017 / Published online: 28 October 2017 \\ (C) The Author(s) 2017. This article is an open access publication
}

\begin{abstract}
Purpose Patients receiving platinum-based chemotherapy are at high risk of chemotherapy-induced nausea and vomiting (CINV), a distressing side effect of treatment. This post-hoc subgroup analysis of two pivotal trials evaluated the efficacy of NEPA in preventing CINV in subsets of patients with lung cancer who received cisplatin or carboplatin.

Methods In each study, the efficacy endpoints complete response (CR; defined as no emetic episodes and no rescue medication) and no significant nausea (NSN; defined as a score of $<25 \mathrm{~mm}$ on a visual analog scale of $0-100 \mathrm{~mm}$ ) during the acute $(0-24 \mathrm{~h})$, delayed $(25-120 \mathrm{~h})$, and overall $(0-120 \mathrm{~h})$ phases post-chemotherapy in cycle 1 (study 1) and cycles 1-4 (study 2) were assessed. Safety was evaluated by recording treatment-emergent adverse events (AEs) and treatment-related AEs.

Results NEPA treatment resulted in high CR rates across the acute, delayed, and overall phases (cisplatin: $>88 \%$ overall CR; carboplatin: $>75 \%$ overall CR), with higher $\mathrm{CR}$ rates for NEPA-treated patients than those receiving palonosetron; moreover, CR rates were sustained over multiple chemotherapy cycles $(>75 \%)$. High rates of NSN observed during cycle $1(>79 \%)$ were also maintained over multiple chemotherapy cycles. NEPA was well tolerated in all patients.
\end{abstract}

Paul J. Hesketh

paul.hesketh@lahey.org

1 Lahey Health Cancer Institute, Lahey Hospital \& Medical Center, 41 Mall Road, Burlington, MA 01805, USA

2 Corporate Clinical Development, Helsinn Healthcare SA, Lugano, Switzerland

3 Helsinn Healthcare SA, Lugano, Switzerland
Conclusions NEPA appears to be effective and well tolerated in patients with lung cancer receiving platinum-based chemotherapy, across the acute, delayed, and overall phases and throughout multiple cycles. As a highly effective oral combination antiemetic agent administered as a single dose once per cycle, NEPA may offer a convenient, simplified prophylactic antiemetic.

Keywords Lung cancer · Cisplatin · Carboplatin .

Chemotherapy-induced nausea and vomiting $(\mathrm{CINV}) \cdot$ NEPA

\section{Introduction}

Lung cancer is the most common cancer worldwide, with an estimated 1.8 million new cases in 2012, comprising nearly $13 \%$ of the total new cancer cases (excluding nonmelanoma skin cancers) diagnosed that year [1]. In the majority of patients with non-small cell lung cancer (NSCLC), and especially those with advanced disease, cytotoxic chemotherapy remains the mainstay of treatment [2-4]. Cisplatin, a highly emetogenic chemotherapeutic, is the gold standard in the adjuvant setting [5, 6], although a less emetogenic carboplatin regimen is the recommended treatment option for patients with comorbidities or who are unable to tolerate cisplatin [7].

One of the most feared side effects of chemotherapy is chemotherapy-induced nausea and vomiting (CINV) [8], which, if poorly controlled, may lead to impaired functioning and quality of life [9], anorexia [6], and potentially postponement or dose reduction of chemotherapy [10]. Recent advances in the development of antiemetic agents and the publication of several evidence-based antiemetic guidelines $[6,11,12]$ have led to vast improvements in the control of CINV, which can now be prevented in $70-80 \%$ of patients [13]. 
To achieve optimal control of CINV, the choice of appropriate antiemetic prophylaxis is paramount. International antiemetic guidelines published by the Multinational Association of Supportive Care in Cancer (MASCC) and the European Society for Medical Oncology (ESMO) [11], the American Society of Clinical Oncology [12], and the National Comprehensive Cancer Network (NCCN) [6] categorize chemotherapy agents according to their emetogenic potential (high, moderate, low, and minimal), and base their recommendations on the level of risk that a chemotherapeutic agent poses. For patients receiving highly emetogenic chemotherapy (HEC), such as cisplatin, the guidelines recommend the triplet combination of a 5-hydroxytryptamine-3 $\left(5-\mathrm{HT}_{3}\right)$ receptor antagonist (RA), a neurokinin-1 $\left(\mathrm{NK}_{1}\right)$ RA, and a corticosteroid, such as dexamethasone $[6,11]$ or a four-drug combination with the addition of olanzapine to the triplet [12]. For patients receiving moderately emetogenic chemotherapy (MEC), the general recommendation for antiemetic prophylaxis is treatment with a $5-\mathrm{HT}_{3} \mathrm{RA}$ in combination with dexamethasone. Although until recently considered MEC, guidelines currently advise that patients receiving carboplatin should be treated with a combination of an $\mathrm{NK}_{1} \mathrm{RA}$, a $5-\mathrm{HT}_{3} \mathrm{RA}$, and dexamethasone $[6,11,12]$, and the NCCN guidelines propose the addition of an $\mathrm{NK}_{1} \mathrm{RA}$ if high-risk factors are present, or if previous $5-\mathrm{HT}_{3} \mathrm{RA}$-dexamethasone treatment has failed [6]. Despite evidence demonstrating that CINV control can be achieved if these recommendations are followed [14], the guidelines are often inadequately adhered to in clinical practice. This may be, in part, due to the perceived complexity of some antiemetic regimens and subsequent poor patient compliance.

NEPA is an oral fixed combination of the highly selective $\mathrm{NK}_{1}$ RA netupitant (NETU; $300 \mathrm{mg}$ ) and the clinically [15] and pharmacologically [16] distinct 5- $\mathrm{HT}_{3} \mathrm{RA}$ palonosetron (PALO; $0.5 \mathrm{mg}$ ) administered as a single dose prior to chemotherapy. The simultaneous targeting of two critical emetic pathways, in concert with the single-dose administration per chemotherapy cycle, results in convenient and long-lasting protection from CINV. Clinical studies have demonstrated the superiority of NEPA plus dexamethasone, compared with PALO plus dexamethasone, in preventing CINV during both the acute and delayed phases in the HEC [17] and anthracyclinecyclophosphamide (AC) settings [18]. In addition, NEPA has been shown to be efficacious over multiple cycles of AC [18] HEC or MEC [19]. Each of these pivotal studies demonstrated the safety and efficacy of NEPA in a population of patients with mixed solid tumors, but did not address any particular patient subgroup.

Herein, we report the results of a post-hoc subgroup analysis from two of the pivotal trials $[17,19]$. The objective was to evaluate the efficacy of NEPA in the subsets of patients with lung cancer who were receiving platinum-based chemotherapy.

\section{Methods}

\section{Trial designs}

The current report is a post-hoc analysis of the subgroup of patients with lung cancer from two published pivotal trials, hereafter referred to as study 1 [17] and study 2 [19]. In both trials, the protocols were approved by the relevant institutional bodies, and each study was followed in accordance with the Declaration of Helsinki principles (2008), International Conference on Harmonization E6 Good Clinical Practice guidelines, as well as national/local laws and regulations.

\section{Study 1}

This was a phase II, multinational, randomized, double-blind, single-cycle, dose-ranging study of oral NEPA versus oral PALO in solid tumors [17]. Patients were scheduled to receive a first dose of cisplatin-based chemotherapy and were stratified by gender and randomized to one of five treatment arms: PALO $0.50 \mathrm{mg}$; NEPA 100 (NETU $100 \mathrm{mg}$ plus PALO $0.50 \mathrm{mg}$ ); $\mathrm{NEPA}_{200}$ (NETU $200 \mathrm{mg}$ plus PALO $0.50 \mathrm{mg}$ ); $\mathrm{NEPA}_{300}$ (NETU $300 \mathrm{mg}$ plus PALO $0.50 \mathrm{mg}$ ); intravenous ondansetron $32 \mathrm{mg}$ plus oral aprepitant, at a dose of $125 \mathrm{mg}$ on day 1 and $80 \mathrm{mg}$ daily on days 2 and 3 (exploratory arm) [17]. All patients also received oral dexamethasone; the PALO arm at a dose of $20 \mathrm{mg}$ on day 1 and $8 \mathrm{mg}$ twice daily on days 2-4; and the NEPA and aprepitant treatment arms at a dose of $12 \mathrm{mg}$ on day 1 and $8 \mathrm{mg}$ daily on days $2-4$.

\section{Study 2}

This phase III, multinational, randomized, double-blind, parallel-group study [19] evaluated NEPA over multiple cycles of chemotherapy. Patients, stratified by HEC/MEC and gender, were randomized in a 3:1 ratio to receive either oral NEPA (NETU $300 \mathrm{mg}$ plus PALO $0.50 \mathrm{mg}$ ) or oral aprepitant $125 \mathrm{mg}$ plus PALO $0.50 \mathrm{mg}$ on day 1 , followed by oral aprepitant $80 \mathrm{mg}$ daily on days 2-3 [19]; the aprepitant arm was included as a reference arm for safety. Patients receiving HEC received oral dexamethasone at a dose of $12 \mathrm{mg}$ on day 1 and $8 \mathrm{mg}$ on days $2-4$, while patients receiving MEC only received oral dexamethasone $12 \mathrm{mg}$ on day 1 .

\section{Lung cancer population}

Eligible patients were aged $\geq 18$ years, diagnosed with any type of lung cancer, chemotherapy naive, and had a Karnofsky 
Table 1 Demographics and baseline characteristics of NEPAtreated patients with lung and non-lung cancers receiving cisplatin or carboplatin chemotherapy and included in the subgroup analyses (safety population

\begin{tabular}{|c|c|c|c|c|}
\hline & \multicolumn{2}{|l|}{$\begin{array}{l}\text { Study } 1 \\
(N=408)\end{array}$} & \multicolumn{2}{|l|}{$\begin{array}{l}\text { Study } 2 \\
(N=216)\end{array}$} \\
\hline & $\begin{array}{l}\text { Lung } \\
\text { cancer } \\
(n=109)\end{array}$ & $\begin{array}{l}\text { Non-lung cancer } \\
(n=299)\end{array}$ & $\begin{array}{l}\text { Lung } \\
\text { cancer } \\
(n=122)\end{array}$ & $\begin{array}{l}\text { Non-lung cancer } \\
(n=94)\end{array}$ \\
\hline \multicolumn{4}{|l|}{ Gender, $n(\%)$} & $53.9 \pm 11.2$ \\
\hline $\begin{array}{l}\text { Male } \\
\text { Female }\end{array}$ & $\begin{array}{l}97(89.0) \\
12(11.0)\end{array}$ & $\begin{array}{l}136(45.5) \\
163(54.5)\end{array}$ & $\begin{array}{l}84(68.9) \\
38(31.1)\end{array}$ & $\begin{array}{l}39(41.5) \\
55(58.5)\end{array}$ \\
\hline \multicolumn{5}{|l|}{ Cancer type, $n(\%)$} \\
\hline $\begin{array}{l}\text { Lung and respiratory tract } \\
\text { cancer }\end{array}$ & $109(100)$ & - & $122(100)$ & - \\
\hline Bladder & - & $14(4.7)$ & - & $4(4.3)$ \\
\hline Breast & - & $26(8.7)$ & - & $7(7.4)$ \\
\hline Colorectal & - & - & - & $1(1.1)$ \\
\hline Gastric & - & $24(8.0)$ & - & $3(3.2)$ \\
\hline Other GI & - & $18(6.0)$ & - & - \\
\hline Gynecologic & - & $97(32.4)$ & - & $42(44.7)$ \\
\hline Other urogenital & - & $9(3.0)$ & - & - \\
\hline Head and neck & - & $91(30.4)$ & - & $20(21.1)$ \\
\hline Rectal & - & - & - & $1(1.1)$ \\
\hline Other & - & $11(3.7)$ & - & $16(17.0)$ \\
\hline $\begin{array}{l}\text { Malignant neoplasm site } \\
\text { unspecified }\end{array}$ & - & $9(3.0)$ & - & - \\
\hline \multicolumn{5}{|c|}{ Karnofsky Performance Status, $n(\%)$} \\
\hline 100 & $7(6.4)$ & $38(12.7)$ & NA & NA \\
\hline 90 & $31(28.4)$ & $204(68.2)$ & NA & NA \\
\hline 80 & $66(60.6)$ & $52(17.4)$ & NA & NA \\
\hline 70 & $5(4.6)$ & $5(1.7)$ & NA & NA \\
\hline \multicolumn{5}{|c|}{ ECOG performance status, $n(\%)$} \\
\hline 0 & NA & NA & $49(40.2)$ & $43(45.7)$ \\
\hline 1 & NA & NA & $70(57.4)$ & $50(53.2)$ \\
\hline 2 & NA & NA & $3(2.5)$ & $1(1.1)$ \\
\hline \multicolumn{5}{|c|}{ Chemotherapy (cycle 1), $n(\%)$} \\
\hline Cisplatin & $109(100)$ & 298 (99.7) & $43(35.2)$ & $29(30.5)$ \\
\hline Carboplatin & - & $1(0.1)$ & $79(64.8)$ & $65(69.1)$ \\
\hline
\end{tabular}

ECOG Eastern Cooperative Oncology Group, GI gastrointestinal, $N A$ not assessed, $S D$ standard deviation
Performance Status (PS) score $\geq 70 \%$ (study 1) or Eastern Cooperative Oncology Group (ECOG) PS 0-2 (study 2).

\section{Assessments}

In each study, the efficacy endpoints of complete response (CR; defined as no emetic episodes and no rescue medication) and no significant nausea (NSN; defined as a visual analog scale score of $<25 \mathrm{~mm}$ on a scale of 0 $100 \mathrm{~mm})$ during the acute $(0-24 \mathrm{~h})$, delayed $(25-120 \mathrm{~h})$, and overall $(0-120 \mathrm{~h})$ phases post-chemotherapy in cycle 1 (study 1 ) and cycles 1-4 (study 2 ) were assessed. Safety was evaluated primarily by treatment-emergent adverse events (TEAEs, occurring after the first dose of study drug) and treatment-related AEs (TRAEs).

\section{Statistical analysis}

The primary aim of this post-hoc analysis was to determine the efficacy of NEPA in preventing CINV in subsets of patients with lung cancer from two pivotal trials [17, 19], who received either cisplatin or carboplatin. The efficacy data were not pooled due to the different comparators and chemotherapy regimens administered in the two trials (study 1: PALO- and cisplatin-based regimen; study 2: aprepitant/PALO and cisplatin- or carboplatinbased regimens). The aprepitant arms of both studies were not included in this analysis, as the arms served either an exploratory or reference purpose, and included small sample sizes. Descriptive statistics for the efficacy parameters were provided for the subpopulation of lung cancer patients receiving NEPA (study 1 and study 2 ) or PALO (study 1). There were no formal statistical comparisons made between the NEPA and PALO arms due to the limited sample size and also because the stratification by gender is lost when performing the subgroup analysis (see "Results" section). In study 1, data from the different NEPA dose groups $(100 / 200 / 300 \mathrm{mg})$ were combined, as all showed similar efficacy in the subgroup of patients with lung cancer. The antiemetic 
Fig. 1 Complete response (CR) rates for lung cancer patients: acute $(0-24 \mathrm{~h})$, delayed $(25-$ $120 \mathrm{~h})$, and overall (0-120 h) CR rates in cycle 1 (efficacy population). a NEPA versus PALO-treated patients receiving cisplatin, study 1. b NEPA-treated patients receiving cisplatin or carboplatin, study 2. NEPA netupitant-palonosetron, $P A L O$ palonosetron a

nEPA $\square$ PALO

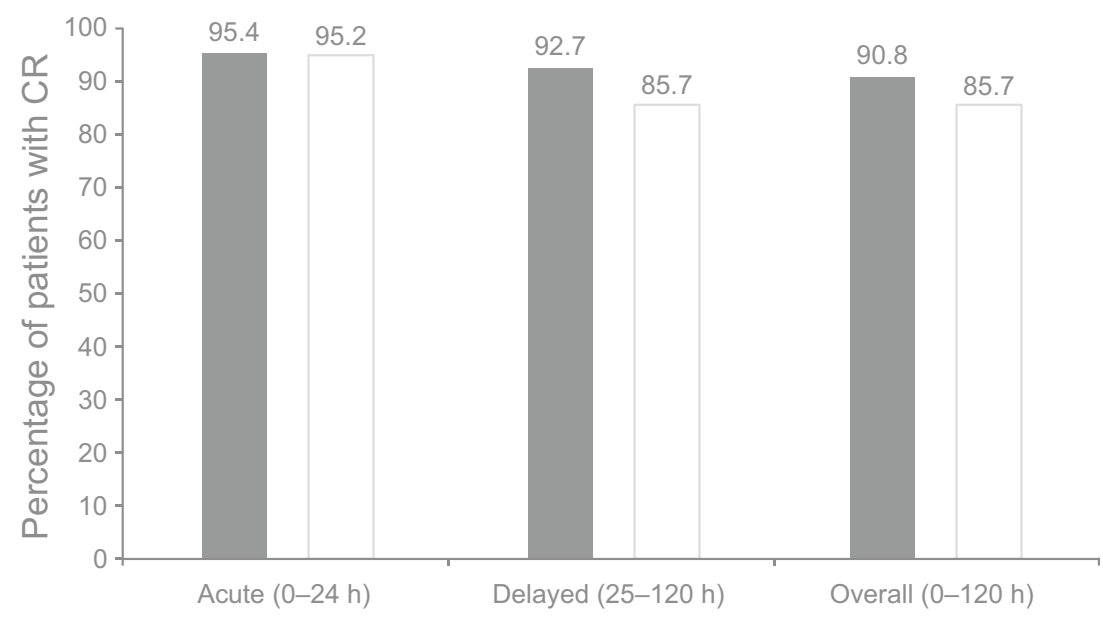

Phase after chemotherapy administration
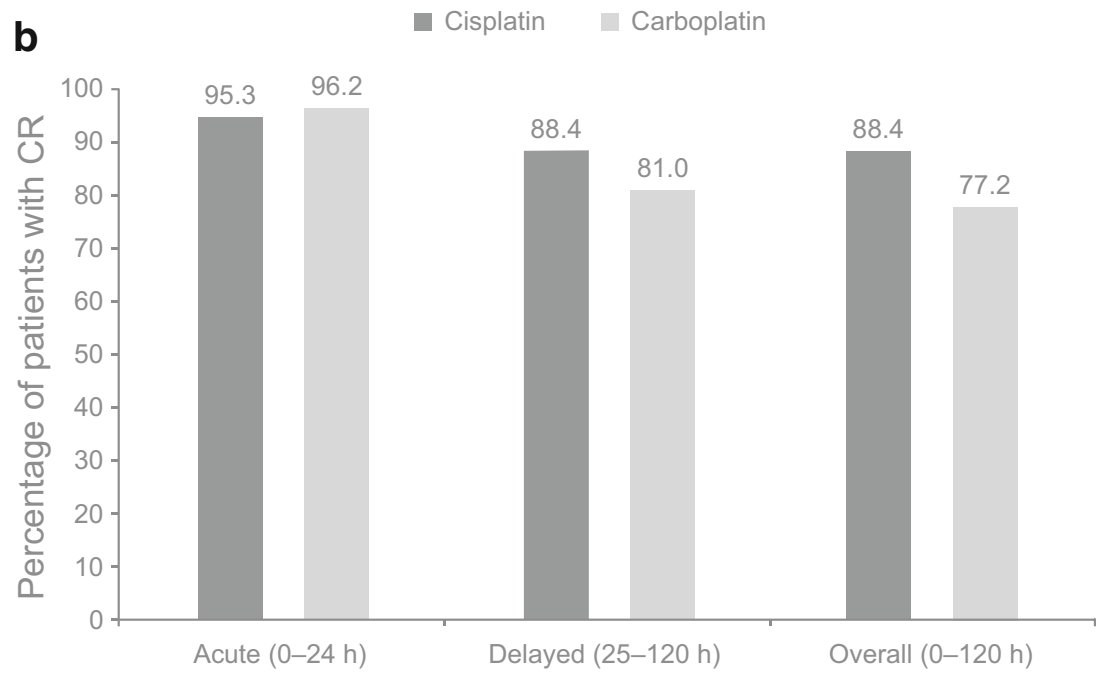

Phase after chemotherapy administration outcomes following platinum-based chemotherapy of the non-lung cancer cohorts were also assessed in both studies to provide a context to interpret the results in the population of patients with lung cancer. Data were presented using descriptive statistics and no formal comparison between the lung cancer and non-lung cancer subsets was performed. All figures displaying demographics, baseline characteristics, and toxicity profile were based on the safety population. In study 1, two patients from the safety population were excluded from the efficacy population: one did not receive chemotherapy (NEPA, lung cancer) and one patient received carboplatin instead of cisplatin (NEPA, non-lung cancer).

\section{Results}

\section{Analyzed patient population}

Of the 694 patients randomized in study 1, 408 received NEPA and platinum-based chemotherapy, and 109 (26.7\%) patients had lung cancer and were included in the safety population. Additionally, in study 1, 136 patients received PALO and chemotherapy, and 42 (30.9\%) of them had lung cancer. In study 2 , of the 413 patients randomized, 216 patients received NEPA and carboplatin or cisplatin, of which 122 (56.5\%) had lung cancer and were included in the safety population.

Overall, this subgroup analysis presented data from 231 NEPA-treated patients with lung cancer and 393 NEPA- 
Fig. 2 Overall $(0-120 \mathrm{~h})$ complete response $(\mathrm{CR})$ rates in NEPA-treated lung cancer patients receiving cisplatin or carboplatin across multiple cycles (study 2) (efficacy population). NEPA netupitant-palonosetron
- Cisplatin Carboplatin

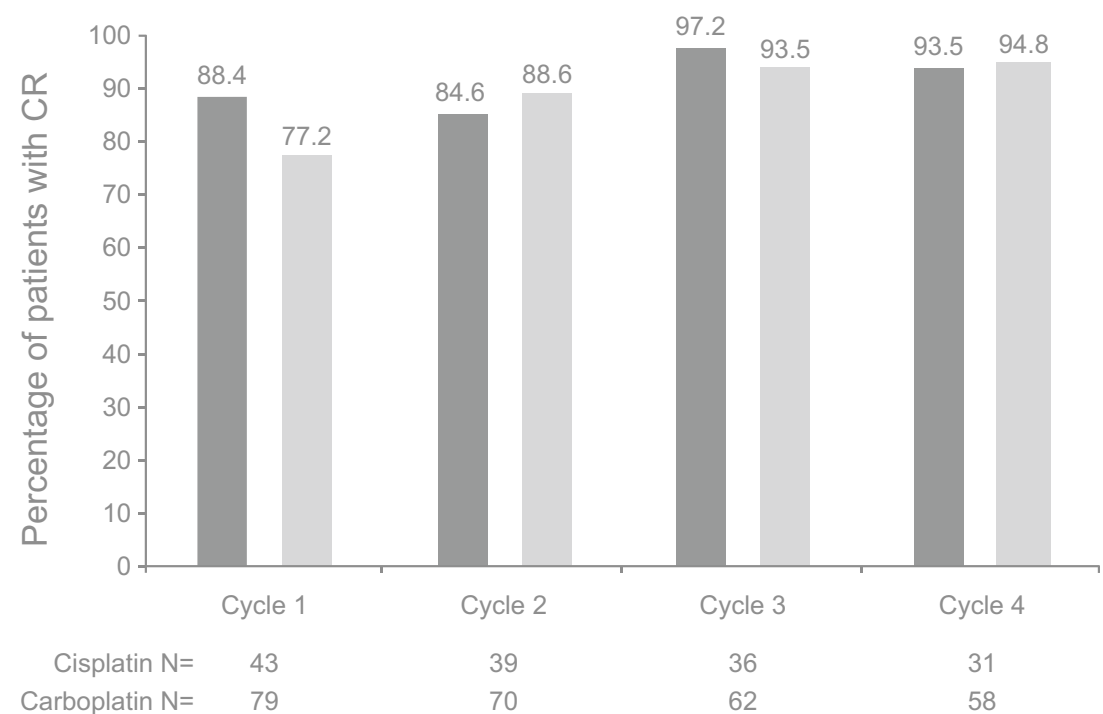

treated patients with non-lung cancers. In study 1 , all patients in the efficacy population $(N=109)$ received a cisplatin-based regimen, while in study 2 , the majority of lung cancer (79/122; $64.8 \%)$ and non-lung cancer $(65 / 94 ; 69.1 \%)$ patients received a carboplatin-based regimen. Demographic and baseline characteristics of the NEPA-treated patients are shown in Table 1 for the safety population. The majority of NEPA-treated lung cancer patients were male (study 1: 89.0\%; study 2: 68.9\%) and had a Karnofsky PS of $80 \%$ (study 1) or ECOG PS of 1 (study 2); the mean age was 56.2 and 60.1 years for study 1 and study 2, respectively. For the non-lung cancer subset, in both studies, there was a greater proportion of females (study 1:54.5\%; study 2: $58.5 \%$ ); the majority had a Karnofsky PS of $90 \%$ (in study 1) and ECOG PS of 1 (study 2); the mean age was 53.9. In both studies, the most common non-lung cancer types were gynecologic and head and neck.

\section{Efficacy: complete response}

In cycle 1, NEPA treatment resulted in high CR rates across the acute, delayed, and overall phases in the subset of lung cancer patients receiving cisplatin- (study 1 and study 2 ; Fig. 1a, b, respectively) or carboplatin-based (study 2; Fig. 1b) chemotherapy. In study 1 , higher $\mathrm{CR}$ rates were observed for NEPA-treated patients compared with PALO-treated patients in the delayed and overall phases. However, comparable CR rates were seen in the acute phase (Fig. 1a). In study 2, overall phase, $\mathrm{CR}$ rates were sustained over repeated cycles in NEPA-treated lung cancer patients receiving either cisplatin or carboplatin (Fig. 2). When considering patients with cancers other than lung, high CR rates were observed regardless of whether patients received cisplatin- (Fig. 3a, b) or carboplatinbased (Fig. 3b) chemotherapy.

\section{Efficacy: no significant nausea}

As was the case for CR, high rates of NSN were observed in the subset of NEPA-treated patients with lung cancer receiving cisplatin or carboplatin during cycle 1 (Table 2). Similar outcomes were seen for PALO-treated patients in study 1. NSN rates were maintained over repeated cycles in NEPA-treated lung cancer patients receiving either cisplatin or carboplatin (Fig. 4). High rates of NSN were also evident in patients with non-lung cancers (Table 2).

\section{Safety}

NEPA was well tolerated in patients with lung and non-lung cancers, regardless of whether cisplatin- or carboplatin-based regimens were administered. In study 1 , asthenia $(7.3 \%)$ and hiccups $(5.5 \%)$ were the only TEAEs reported in $\geq 5 \%$ of patients with lung cancer; the most common TRAE was hiccups $(5.5 \%)$. In lung cancer patients from study 2 - cycle 1 , the most frequently reported TEAEs in $\geq 5 \%$ of patients were neutropenia (21.3\%), leukopenia (14.8\%), anemia (10.7\%), and alopecia (9.0\%); headache (1.6\%) was the most common TRAE. Overall, for the lung cancer subset, the only TRAEs occurring in $\geq 2 \%$ of the patients in either study were hiccups.

For the non-lung cancer subset, the most common TEAEs in study 1 were asthenia and leukocytosis (6.7\% each), headache $(6.4 \%)$, alanine aminotransferase increase $(6.0 \%)$, and dyspepsia (5.4\%); in study 2-cycle 1, the most common were leukopenia $(17.0 \%)$, neutropenia $(14.9 \%)$, alopecia (13.8\%), anemia and pyrexia (8.5\% each), cancer pain, constipation, dyspepsia, headache, and thrombocytopenia (5.3\% each). Regarding TRAEs, the most frequently reported in 
Fig. 3 Complete response (CR) rates for NEPA-treated patients with lung cancer versus those with non-lung cancer: acute ( 0 $24 \mathrm{~h})$, delayed $(25-120 \mathrm{~h})$, and overall $(0-120 \mathrm{~h}) \mathrm{CR}$ rates in cycle 1 (efficacy population). a Patients receiving cisplatin, study 1. b Patients receiving cisplatin or carboplatin, study 2. NEPA netupitant-palonosetron a

- Lung cancer (cisplatin; $N=109)$ Non-lung cancer (cisplatin; $N=298$ )

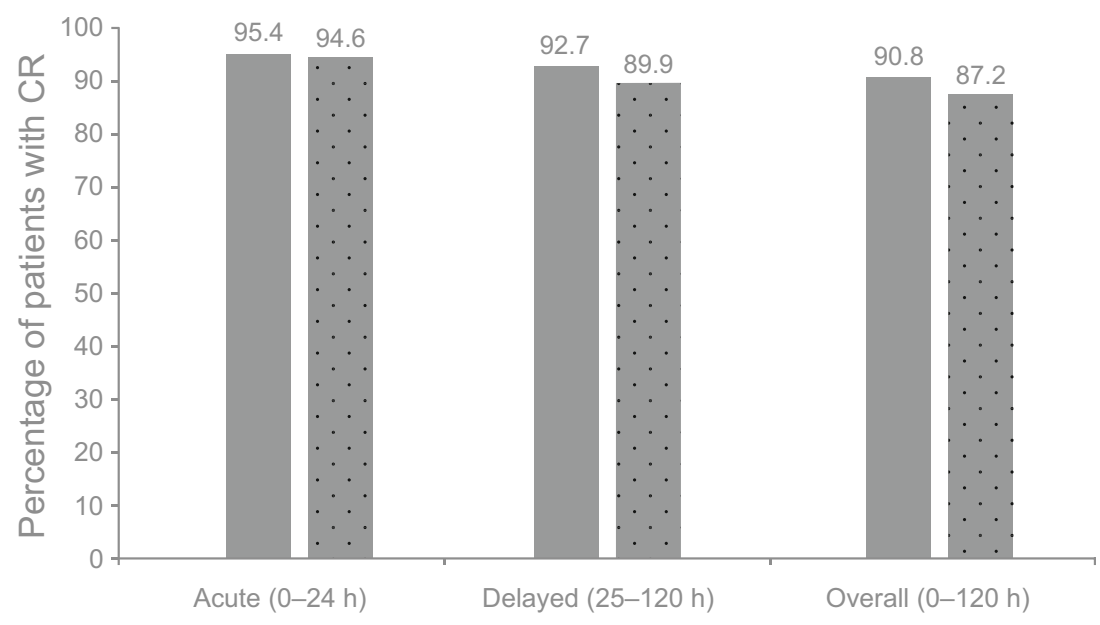

Phase after chemotherapy administration

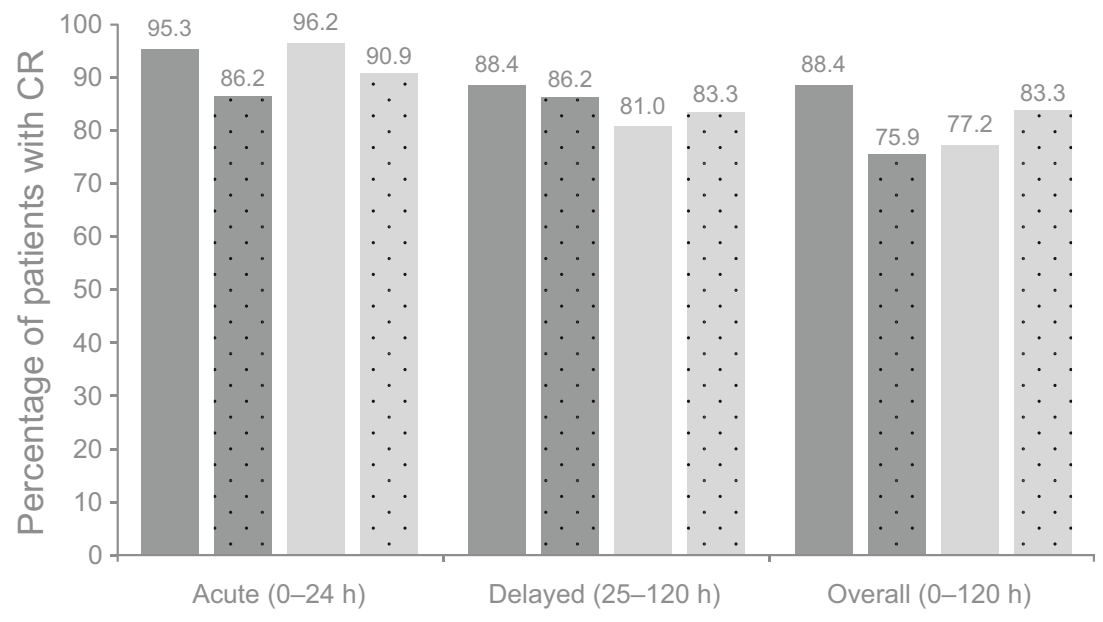

Phase after chemotherapy administration

study 1 were hiccups $(3.7 \%)$ and, in study 2 - cycle 1 , constipation $(2.1 \%)$.

\section{Discussion}

Pivotal trials in patients with a wide range of solid tumors have shown that NEPA, the first combination antiemetic, is superior to PALO in preventing CINV $[17,18]$, and maintained high CR rates across multiple cycles of chemotherapy, with a small but consistent numeric advantage over aprepitant plus PALO [19]. Herein, we report the safety and efficacy results of a post-hoc analysis of the subgroups of patients with lung cancer from two pivotal trials [17, 19]. In study 1 [17], patients received a cisplatin-based regimen, while in study 2 [19], patients received a regimen containing either cisplatin or carboplatin, two agents that are commonly used to treat lung cancer [7]. While cisplatin is well-established as the most highly emetogenic chemotherapeutic, carboplatin is at the high end of the MEC category, with recent antiemetic guidelines now recommending that patients receiving carboplatin 
Table 2 No significant nausea (NSN) rates in NEPA-treated lung and non-lung cancer patients receiving cisplatin or carboplatin in cycle 1 (efficacy population)

\begin{tabular}{|c|c|c|c|c|c|c|c|}
\hline & Study 1 & & & Study 2 & & & \\
\hline \multirow[t]{3}{*}{ NSN, \% } & Cisplatin & & & Cisplatin & & Carboplatin & \\
\hline & Lung cancer & & Non-lung cancer & Lung cancer & Non-lung cancer & Lung cancer & Non-lung cancer \\
\hline & $\begin{array}{c}\text { NEPA } \\
(n=109)\end{array}$ & $\begin{array}{c}\text { PALO } \\
(n=42)\end{array}$ & $\begin{array}{c}\text { NEPA } \\
(n=298)\end{array}$ & $\begin{array}{c}\text { NEPA } \\
(n=43)\end{array}$ & $\begin{array}{c}\text { NEPA } \\
(n=29)\end{array}$ & $\begin{array}{c}\text { NEPA } \\
(n=79)\end{array}$ & $\begin{array}{c}\text { NEPA } \\
(n=66)\end{array}$ \\
\hline Acute $(0-24 \mathrm{~h})$ phase & 95.4 & 95.2 & 95.6 & 90.7 & 93.1 & 83.5 & 93.9 \\
\hline Delayed (25-120 h) phase & 89.0 & 88.1 & 86.6 & 81.4 & 86.2 & 79.7 & 90.9 \\
\hline Overall $(0-120 \mathrm{~h})$ phase & 87.2 & 88.1 & 84.6 & 81.4 & 82.8 & 79.7 & 89.4 \\
\hline
\end{tabular}

NEPA netupitant-palonosetron, $P A L O$ palonosetron

be given a prophylactic triplet antiemetic combination of an $\mathrm{NK}_{1} \mathrm{RA}$, a 5-HT 3 RA, and dexamethasone $[6,11,12]$.

In this report, NEPA appears to be effective in preventing nausea and vomiting in the subgroup of patients with lung cancer. High CR rates were observed across the acute, delayed, and overall phases regardless of whether cisplatin- or carboplatin-based chemotherapy was administered. As expected, the highest $\mathrm{CR}$ rates were seen in the acute phases in both studies, although NEPA was also efficacious in the delayed and overall phases, with sustained efficacy demonstrated across multiple cycles. In study 1 , numerically higher CR rates were observed in NEPA-treated patients with lung cancer, compared with those who received PALO, mirroring the results in the original study [17], although no formal statistical comparisons were undertaken in this subgroup analysis. Of note, in study 2 [19] the CR rates observed in cycle 1 were higher in the delayed and overall phases for lung cancer patients receiving cisplatin than those patients receiving carboplatin. These findings are somewhat surprising given the respective emetogenic potential of cisplatin and carboplatin. However, as there were no appreciable differences between the baseline characteristics or potential CINV risk factors of patients receiving cisplatin or carboplatin, it is most likely that this observation was an artefact of the small sample size.

Consistent with the CR results, high NSN rates were observed in NEPA-treated lung cancer patients receiving either carboplatin or cisplatin. In study 1, NSN rates were surprisingly similar for NEPA and PALO treatment groups across all phases. However, the lack of a difference during the delayed/ overall phases ( 89.0 vs $88.1 \%$ for NEPA and PALO, respectively, in the delayed phase, and 87.2 and $88.1 \%$ for NEPA and PALO, respectively, in the overall phase) appeared to be due to the higher-than-expected efficacy seen with PALO rather than with NEPA, as the NSN rates for NEPA in lung cancer patients mimicked those seen in the overall study population [17], while the rates for PALO were approximately $8 \%$ higher in the lung cancer subset than in the overall study population
Fig. 4 Overall $(0-120 \mathrm{~h})$ no significant nausea (NSN) rates in NEPA-treated lung cancer patients receiving cisplatin or carboplatin across multiple cycles (study 2) (efficacy population). NEPA netupitant-palonosetron
Cisplatin Carboplatin

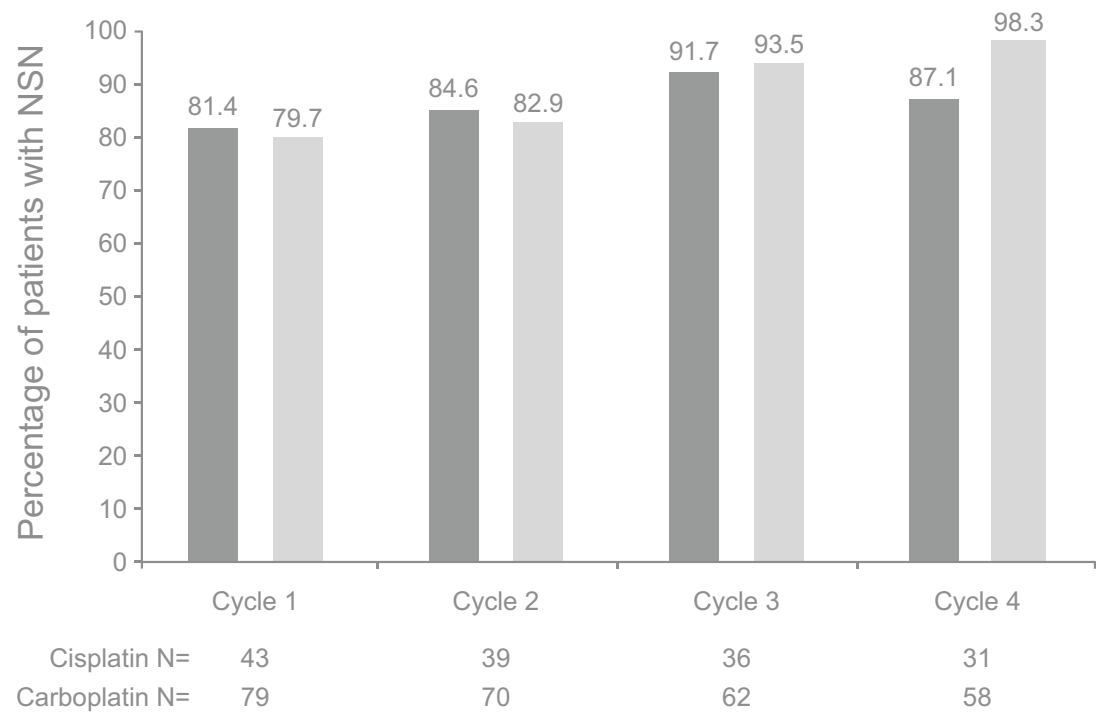


[17]. This may simply be a function of the small sample size $(N=42)$ in the PALO subset.

PALO, a second-generation 5- $\mathrm{HT}_{3} \mathrm{RA}$, has been shown to be more effective than older $5-\mathrm{HT}_{3}$ RAs for the control of CINV in the delayed and overall phases [20]. Included both as a component of the fixed combination NEPA, and in the aprepitant-PALO-dexamethasone reference regimen, PALO may have contributed to the high antiemetic efficacy observed in both studies.

Considering the subsets of patients with non-lung cancers, the data suggest that NEPA is at least as effective in lung cancer patients as those with other cancers. In NEPA-treated non-lung cancer patients, high rates of CR and NSN were observed, with slightly better control achieved in patients receiving carboplatin than may be anticipated.

NEPA was also well tolerated in both studies in the lung and non-lung cancer populations, with AEs typical of a population of cancer patients undergoing emetogenic chemotherapy.

Limitations to the present study include its exploratory nature, given it is a post-hoc analysis, as well as the lack of formal statistical comparisons. Recent developments in the field of CINV have led to the approval of new drugs that have been rapidly incorporated in guidelines recommendations. Due to its post-hoc nature, the comparator arms included in this subgroup analysis do not correspond with current guidelines recommendations $[6,11,12]$. Factors that preclude the ability to perform formal statistical analyses between study arms (NEPA vs PALO) and patient subgroups (lung cancer vs non-lung cancer) include (1) the limited sample size of the lung cancer population in both studies, (2) non-balanced patient demographics between the different subgroups due to the loss of patient stratification by gender for the analysis, and (3) further division of the subgroups according to the anticancer treatment received (cisplatin or carboplatin) resulting in a further reduction of the sample size.

The data reported herein are not dissimilar to results from a number of studies examining the efficacy of the aprepitantPALO-dexamethasone triplet regimen in preventing CINV in patients with lung cancer. One study reported overall $\mathrm{CR}$ rates in cisplatin- $(71 \%)$ and carboplatin-treated patients $(86 \%)$ that are similar to those reported in the current study [21]. Two other studies evaluating the efficacy of the aprepitant-PALOdexamethasone combination in patients with NSCLC receiving a carboplatin-based regimen [22, 23] also reported CR rates in the overall phase (83.8\% [22] and 80.5\% [23]) similar to those reported herein $(77.2 \%)$. Finally, there is at least one report that has assessed the aprepitant-PALO-dexamethasone antiemetic triplet in lung cancer patients receiving multiple cycles of cisplatin-based chemotherapy [24]. In that study, the reported CR rates in the overall phase increased from $74.4 \%$ (cycle 1) to $78.6 \%$ (cycle 6), which are slightly lower than those reported herein, where the overall CR rate was $88.4 \%$ in cycle 1 and increased to $93.5 \%$ by cycle 4 for cisplatin-treated patients in study 2 . The above-mentioned limitations of the present study may help explain the differences in the results.

In conclusion, the data from this post-hoc analysis of the subgroup of patients with lung cancer from two pivotal trials are in alignment with the results reported in the parent studies $[17,19]$. NEPA, the first oral combination antiemetic, has been shown to be safe and effective in patients with lung cancer receiving platinum-based chemotherapy across the acute, delayed, and overall phases and throughout multiple cycles. As an oral treatment targeting two antiemetic pathways with a single dose administered only once per cycle, NEPA offers a convenient and simplified prophylactic option. Consequently, this drug may facilitate adherence to antiemetic guidelines and improve treatment compliance, which in turn could lead to improved CINV prevention.

Acknowledgments The authors thank patients, investigators, and the study teams. The trials described within this paper were sponsored by Helsinn Healthcare SA, Lugano, Switzerland. The authors would also like to acknowledge and thank Elio Carreras for his involvement in some of the statistical analyses of these studies. Editorial and medical writing assistance was provided by Joanne Franklin, PhD, CMPP, TRM Oncology, The Hague, the Netherlands, and funded by Helsinn Healthcare SA, Lugano, Switzerland.

\section{Compliance with ethical standards}

Data Authors have full control of all primary data and agree that the journal may review the data if requested.

Conflict of interest PJH has no conflicts of interest to declare. MP and PN are employees of Helsinn and may hold stock.

Statement of human rights For this type of study, formal consent is not required.

Statement on the welfare of animals This article does not contain any studies with animals performed by any of the authors.

Statement on informed consent Informed consent was obtained from all individual participants included in the study.

Open Access This article is distributed under the terms of the Creative Commons Attribution-NonCommercial 4.0 International License (http:// creativecommons.org/licenses/by-nc/4.0/), which permits any noncommercial use, distribution, and reproduction in any medium, provided you give appropriate credit to the original author(s) and the source, provide a link to the Creative Commons license, and indicate if changes were made.

\section{References}

1. International Agency for Research on Cancer (2014) GLOBOCAN 2012 v1.0. Cancer incidence and mortality worldwide: IARC CancerBase No. 11. http://globocan.iarc.fr. Accessed 27 Jan 2017

2. Sörenson S, Glimelius B, Nygren $P$ on behalf of the SBU-group (2001) Swedish Council of Technology Assessment in health care. 
A systematic overview of chemotherapy effects in nonsmall cell lung cancer. Acta Oncol 40:327-339

3. Goffin J, Lacchetti C, Ellis PM, Ung YC, Evans WK (2010) Firstline systemic chemotherapy in the treatment of advanced non-small cell lung cancer: a systematic review. J Thorac Oncol 5:260-274

4. Evans TL (2015) Chemotherapy in advanced non-small cell lung cancer: optimal treatment approach for elderly and patients with poor performance status. Am J Hematol Oncol 11:12-16

5. Dong X, Huang J, Cao R, Li L (2010) Palonosetron for prevention of acute and delayed nausea and vomiting in non-small-cell lung carcinoma patients. Med Oncol 28:1425-1429

6. National Comprehensive Cancer Network, NCCN clinical practice guidelines in oncology (NCCN Guidelines $\left.{ }^{\circledR}\right)$, Antiemesis version 2.2017. https://www.nccn.org/professionals/physician_gls/pdf/ antiemesis.pdf. Accessed August 2017

7. National Comprehensive Cancer Network (2016) NCCN clinical practice guidelines in oncology. Non-small cell lung cancer version 4.2016. https://www.ncen.org/professionals/physician gls/pdf/ nscl.pdf. Accessed 27 Jan 2017

8. Griffin AM, Butow PN, Coates AS, Childs AM, Ellis PM, Dunn SM, Tattersall MH (1996) On the receiving end. V: patient perceptions of the side effects of cancer chemotherapy in 1993. Ann Oncol 7:189-195

9. Cohen L, de Moor CA, Eisenberg P, Ming EE, Hu H (2007) Chemotherapy-induced nausea and vomiting: incidence and impact on patient quality of life at community oncology settings. Support Care Cancer 15:497-503

10. Viale PH, Grande C, Moore S (2012) Efficacy and cost: avoiding undertreatment of chemotherapy-induced nausea and vomiting. Clin J Oncol Nurs 16:E133-E141

11. Roila F, Molassiotis A, Herrstedt J et al (2016) MASCC and ESMO guideline update for the prevention of chemotherapy- and radiotherapy-induced nausea and vomiting and of nausea and vomiting in advanced cancer patients. Ann Oncol 2016;27:v119 $-\mathrm{v} 133$

12. Hesketh PJ, Kris MG, Basch E et al (2017) Antiemetics: American Society of Clinical Oncology clinical practice guideline update. J Clin Oncol. 35:3240-3261

13. Jordan K, Sippel C, Schmoll HJ (2007) Guidelines for antiemetic treatment of chemotherapy-induced nausea and vomiting: past, present, and future recommendations. Oncologist 12:1143-1150

14. Aapro M, Molassiotis A, Dicato M, Peláez I, Rodriguez-Lescure Á, Pastorello D, Ma L, Gu A, Gascon P, Roila F, PEER investigators (2012) The effect of guideline-consistent antiemetic therapy on chemotherapy-induced nausea and vomiting (CINV): the pan European emesis registry (PEER). Ann Oncol 23:1986-1992

15. Jordan K, Jahn F, Aapro M (2015) Recent developments in the prevention of chemotherapy-induced nausea and vomiting (CINV): a comprehensive review. Ann Oncol 26:1081-1090
16. Rojas C, Raje M, Tsukamoto T, Slusher BS (2014) Molecular mechanisms of 5-HT(3) and $\mathrm{NK}(1)$ receptor antagonists in prevention of emesis. Eur J Pharmacol 722:26-37

17. Hesketh PJ, Rossi G, Rizzi G, Palmas Alyasova A, Bondarenko I, Lisyanskaya A, Gralla RJ (2014) Efficacy and safety of NEPA, an oral combination of netupitant and palonosetron, for prevention of chemotherapy-induced nausea and vomiting following highly emetogenic chemotherapy: a randomized dose-ranging pivotal study. Ann Oncol 25:1340-1346

18. Aapro M, Rugo H, Rossi G, Rizzi G, Borroni ME, Bondarenko I, Sarosiek T, Oprean C, Cardona-Huerta S, Lorusso V, Karthaus M, Schwartzberg L, Grunberg S (2014) A randomized phase III study evaluating the efficacy and safety of NEPA, a fixed-dose combination of netupitant and palonosetron, for prevention of chemotherapy-induced nausea and vomiting following moderately emetogenic chemotherapy. Ann Oncol 25:1328-1333

19. Gralla RJ, Bosnjak SM, Hontsa A, Balser C, Rizzi G, Rossi G, Borroni ME, Jordan K (2014) A phase III study evaluating the safety and efficacy of NEPA, a fixed-dose combination of netupitant and palonosetron, for prevention of chemotherapyinduced nausea and vomiting over repeated cycles of chemotherapy. Ann Oncol 25:1333-1339

20. Schwartzberg L, Barbour SY, Morrow GR, Ballinari G, Thorn MD, Cox D (2014) Pooled analysis of phase III clinical studies of palonosetron versus ondansetron, dolasetron, and granisetron in the prevention of chemotherapy-induced nausea and vomiting (CINV). Support Care Cancer 22:469-477

21. Kitazaki T, Fukuda Y, Fukahori S, Oyanagi K, Soda H, Nakamura Y, Kohno S (2014) Usefulness of antiemetic therapy with aprepitant, palonosetron, and dexamethasone for lung cancer patients on cisplatin-based or carboplatin-based chemotherapy. Cancer Chemother Pharmacol 73:1129-1136

22. Ito Y, Karayama M, Inui N, Kuroishi S, Nakano H, Nakamura Y, Yokomura K, Toyoshima M, Shirai T, Masuda M, Yamada T, Yasuda K, Hayakawa H, Suda T, Chida K (2014) Aprepitant in patients with advanced non-small-cell lung cancer receiving carboplatin-based chemotherapy. Lung Cancer 84:259-264

23. Kusagaya H, Inui N, Karayama M, Fujisawa T, Enomoto N, Kuroishi S, Nakamura Y, Matsua H, Yokomura K, Koshimizu N, Toyoshima M, Imokawa S, Yamada T, Shirai T, Hayakawa H, Suda $\mathrm{T}$ (2015) Evaluation of palonosetron and dexamethasone with or without aprepitant to prevent carboplatin-induced nausea and vomiting in patients with advanced non-small-cell lung cancer. Lung Cancer 90:410-416

24. Longo F, Mansueto G, Lapadula V, Stumbo L, Del Bene G, Adua D, De Filippis L, Bonizzoni E, Quadrini S (2012) Combination of aprepitant, palonosetron and dexamethasone as antiemetic prophylaxis in lung cancer patients receiving multiple cycles of cisplatinbased chemotherapy. Int J Clin Pract 66:753-757 